Open Access

\title{
Cutaneous exposure to agglomerates of silica nanoparticles and allergen results in lgE-biased immune response and increased sensitivity to anaphylaxis in mice
}

Toshiro Hirai ${ }^{1}$, Yasuo Yoshioka ${ }^{1,2,3^{*}}$, Hideki Takahashi ${ }^{1,2}$, Ko-ichi Ichihashi ${ }^{1}$, Asako Udaka ${ }^{1}$, Takahide Mori ${ }^{4}$, Nobuo Nishijima', Tokuyuki Yoshida', Kazuya Nagano ${ }^{5}$, Haruhiko Kamada5,6, Shin-ichi Tsunoda ${ }^{5,6}$, Tatsuya Takagi ${ }^{7,8}$, Ken J. Ishii ${ }^{9,10}$, Hiromi Nabeshi ${ }^{11}$, Tomoaki Yoshikawa' ${ }^{1}$, Kazuma Higashisaka ${ }^{1,5}$ and Yasuo Tsutsumi ${ }^{1,4,6^{*}}$

\begin{abstract}
Background: The skin is a key route of human exposure to nanomaterials, which typically occurs simultaneously with exposure to other chemical and environmental allergen. However, little is known about the hazards of nanomaterial exposure via the skin, particularly when accompanied by exposure to other substances.

Results: Repeated topical treatment of both ears and the shaved upper back of NC/Nga mice, which are models for human atopic dermatitis (AD), with a mixture of mite extract and silica nanoparticles induced AD-like skin lesions. Measurements of ear thickness and histologic analyses revealed that cutaneous exposure to silica nanoparticles did not aggravate AD-like skin lesions. Instead, concurrent cutaneous exposure to mite allergens and silica nanoparticles resulted in the low-level production of allergen-specific lgGs, including both the Th2-related IgG1 and Th1-related IgG2a subtypes, with few changes in allergen-specific IgE concentrations and in Th1 and Th2 immune responses. In addition, these changes in immune responses increased the sensitivity to anaphylaxis. Low-level lgG production was induced when the mice were exposed to allergen-silica nanoparticle agglomerates but not when the mice exposed to nanoparticles applied separately from the allergen or to well-dispersed nanoparticles.

Conclusions: Our data suggest that silica nanoparticles themselves do not directly affect the allergen-specific immune response after concurrent topical application of nanoparticles and allergen. However, when present in allergenadsorbed agglomerates, silica nanoparticles led to a low lgG/lgE ratio, a key risk factor of human atopic allergies. We suggest that minimizing interactions between nanomaterials and allergens will increase the safety of nanomaterials applied to skin.
\end{abstract}

Keywords: Atopic dermatitis, Agglomerate, Aggregate, Anaphylaxis, Blocking antibody, IgE, IgG, Mite, Nanomaterials, Nanoparticles, Particulate matter

\footnotetext{
* Correspondence: y-yoshioka@biken.osaka-u.ac.jp; ytsutsumi@phs.osaka-u.ac.jp

${ }^{1}$ Laboratory of Toxicology and Safety Science, Graduate School of

Pharmaceutical Sciences, Osaka University, 1-6 Yamadaoka, Suita, Osaka

565-0871, Japan

Full list of author information is available at the end of the article
} 


\section{Introduction}

Because of their unique features and physicochemical properties [1, 2], nanomaterials are increasingly being used to add value to new and existing goods, such as cosmetics, foods, medicines, and industrial products [3-5]. However, these same novel features of nanomaterials make them hazardous under some conditions [6, 7]. To take full advantage of the potential benefits of nanomaterials, we must learn more about their hazards so that safer nanomaterials can be designed.

Numerous epidemiologic studies have prompted concerns regarding the health risks associated with exposure to nanomaterials; in particular, such studies have revealed that exposure to particulate matter (PM), including PM2.5 and Asian dust, induces many adverse effects, including facilitating the onset and severity of allergic diseases [8-11]. Because inhalational exposure to PM has been considered to be the main inducer of these adverse effects, this route has received the most attention regarding exposure to nanomaterials [12]. However, the skin is a major route of both intentional (from clothing, cosmetics, and other skin care products) and unintentional (from the environment) exposure to nanomaterials [13-15]. Furthermore, exposure to nanomaterials often occurs simultaneously with exposure to other chemical compounds and environmental allergens [16], but little is known about the hazards of cutaneous exposure to nanomaterials, particularly in combination with other substances.

In the current study, we investigated the effects of concurrent topical application of mite extract and amorphous silica nanoparticles, one of the most widely used nanomaterials, on allergic sensitization and AD in NC/Nga mice, a murine model of AD. We found that cutaneous exposure to the allergen and silica nanoparticles simultaneously did not aggravate AD-like skin lesions in the mice but resulted in low-level IgG production with little change in IgE production (IgE-biased immune response) and increased sensitivity to anaphylaxis. We suggest that an IgEbiased immune response was induced independently of the innate biologic effects of silica nanoparticles. Because a low $\operatorname{IgG/IgE~ratio~is~a~characteristic~feature~of~human~}$ atopic allergy, we believe that minimizing interactions between nanomaterials and allergens may improve the safety of cutaneously applied nanomaterials.

\section{Results and discussion}

Effects of co-exposure to mite allergen and silica nanoparticles in a murine model of $A D$

For these experiments, we used silica nanoparticles with a diameter of $30 \mathrm{~nm}$ (nSP30). Solutions of nSP30 were clear and colorless (Fig. 1a), and transmission electron microscopy (TEM) revealed that the particles were smooth spheres (Fig. 1b). The size distribution spectrum of nSP30 was a single peak (Fig. 1c), and the mean hydrodynamic diameter $(24.1 \mathrm{~nm}$, as measured by means of a dynamic light-scattering method; Fig. 1d) corresponded almost exactly to the primary particle size. These results indicate that the nSP30 particles were well dispersed in solution.

To examine the effects of co-exposure of skin to allergen and nSP30, we used an extract of the mite Dermatophagoides pteronyssinus (Dp) and NC/Nga mice as a model for human AD [17]. Dp is a frequent cause of many allergic conditions, including asthma and $\operatorname{AD}[18,19]$. In addition, NC/Nga mice have a genetic skin barrier defect related to low ceramide production [20]. To induce ADlike skin lesions, we repeatedly cutaneously exposed NC/ Nga mice to either Dp alone or a mixture of $\mathrm{Dp}$ and nSP30 in an isotonic solution (phosphate buffered saline; PBS). Note that although the solutions of Dp alone and nSP30 alone were clear and colorless, the mixture of Dp + nSP30 was cloudy (Fig. 1a). TEM images suggested that mixing resulted in the formation of agglomerates (Fig. 1b), which was confirmed by the fact that the mean hydrodynamic diameter of the particles in the mixture was $1310.0 \mathrm{~nm}$, which was larger than that of nSP30 alone (Fig. 1c and d). First, we confirmed that exposure to nSP30 alone did not induce the formation of topical skin lesions (Additional file 1). Comparison of the PBS and Dp-alone groups indicated that cutaneous exposure to Dp induced ear thickening, scab formation, acanthosis, inflammatory cell infiltration, and mast cell infiltration (Fig. 2a-e). The effects of cutaneous exposure to Dp + nSP30 did not differ from those of Dp alone, except that the extent of ear thickening was slightly less in the $\mathrm{Dp}+\mathrm{nSP} 30$ group than in the Dp-alone group.

Total IgE levels in plasma, which are often elevated in $\mathrm{AD}$ and other allergic conditions [21], were measured $24 \mathrm{~h}$ after the final treatment. The total IgE level in the Dp-alone group was higher than that in the PBS group (Fig. 2f), and the total IgE level in the Dp + nSP30 group was slightly higher than that in the $\mathrm{Dp}$-alone group (Fig. 2f). Because a Th2-mediated immune response including IgE production is unnecessary for the development of AD-like skin lesions in NC/Nga mice [22], the high total IgE level induced by cutaneous exposure to $\mathrm{Dp}+\mathrm{nSP} 30$ likely did not exacerbate the Dp-induced AD-like skin lesions in NC/Nga mice.

\section{Effect of cutaneous exposure to Dp + nSP30 on cutaneous allergic sensitization}

To clarify the effect of topical Dp + nSP30 on cutaneous allergic sensitization, we evaluated the systemic immune responses $24 \mathrm{~h}$ after the final treatment. Although Dpspecific IgE levels were higher in both the Dp-alone group and the $\mathrm{Dp}+\mathrm{nSP} 30$ group than in the PBS group, Dp-specific IgE levels did not differ significantly between the Dp-alone group and the Dp + nSP30 group (Fig. 3a). 


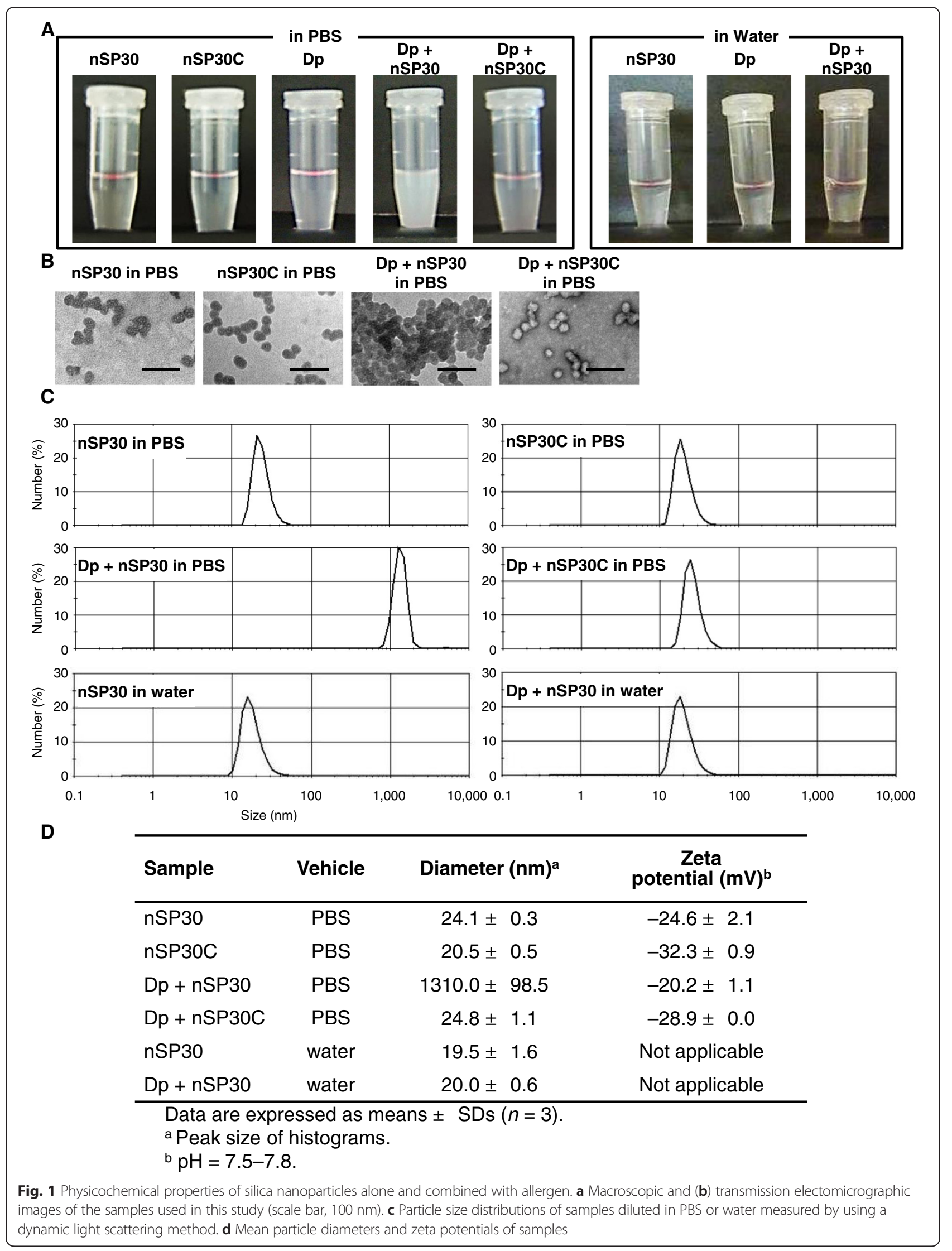




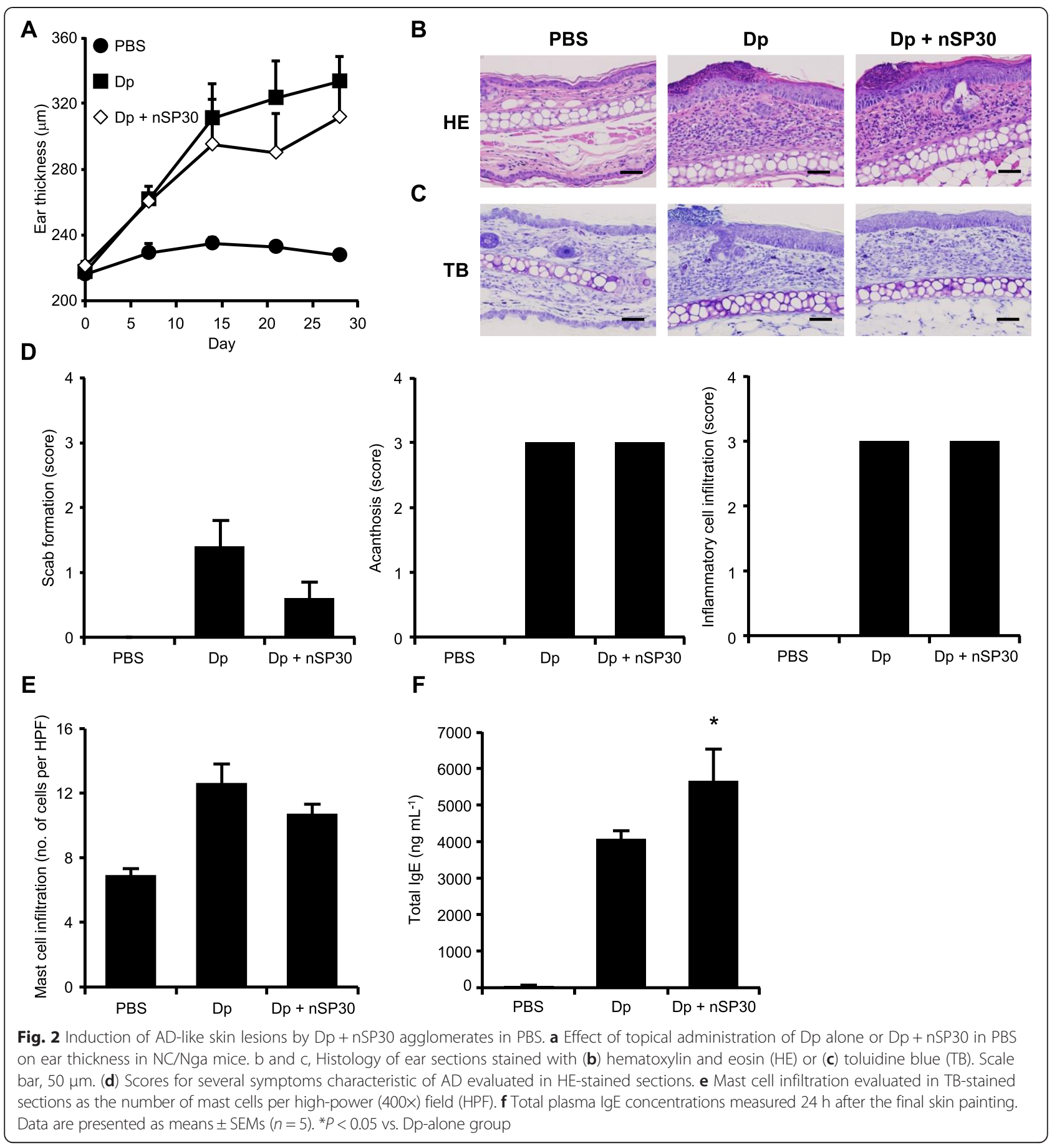

In contrast, the levels of Dp-specific total IgG and all evaluated IgG subtypes were significantly lower in the $\mathrm{Dp}+\mathrm{nSP} 30$ group than in the Dp-alone group (Fig. 3b and c). In addition, we confirmed that nSP30 dosedependently suppressed IgG production (Additional file 2 ). IgG subclass responses have been used to assess the type of immune response; IgG1 is known to indicate a Th2-type response, whereas IgG2a indicates a Th1 response [23]. Thus it is possible that skin exposure to
Dp + nSP30 suppressed both the Th1 and the Th2 responses.

To further characterize the systemic immune responses, we enumerated the Dp-specific splenocytes secreting interferon- $\gamma$ (IFN- $\gamma$ ) and interleukin-4 (IL-4) in each mouse by using cytokine-specific enzyme-linked immunosorbent spot (ELISPOT) assays (Fig. 3d). The numbers of Dp-specific IFN- $\gamma$ - and IL-4-secreting splenocytes did not differ between the Dp-alone group and the Dp + nSP30 

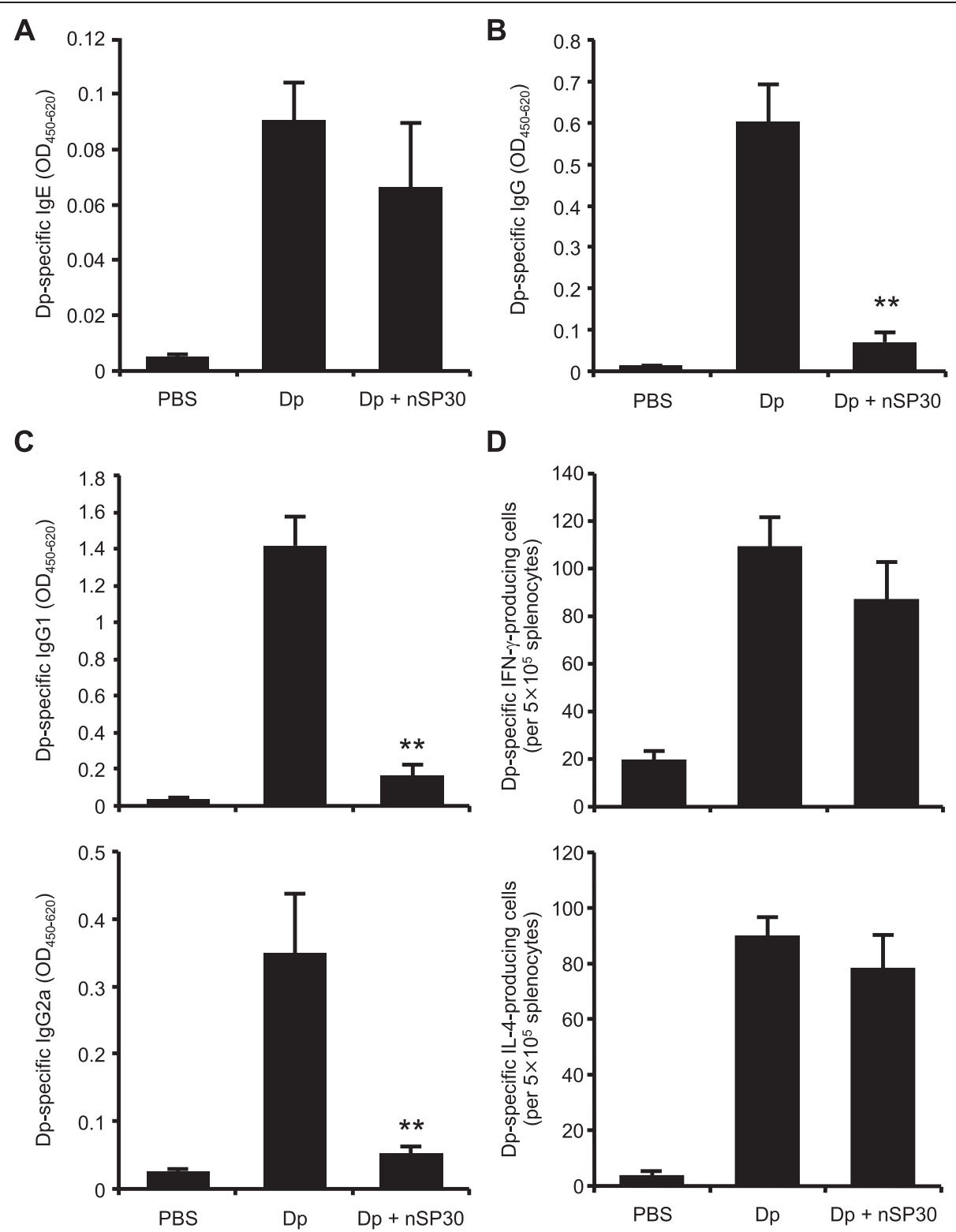

Fig. 3 Induction of systemic immune responses by Dp + nSP30 agglomerates in PBS. a-c Plasma levels of Dp-specific (a) lgE, (b) IgG, and (c) IgG1 and IgG2a as analyzed by ELISA at $24 \mathrm{~h}$ after final topical treatment of NC/Nga mice with Dp alone or Dp + nSP30 in PBS. (D) Numbers of Dp-specific IFN- $\gamma$ - and IL-4-producing splenocytes after re-stimulation with $100 \mu \mathrm{g} \mathrm{mL}{ }^{-1} \mathrm{Dp}$, as determined by using ELISPOT assays specific for each cytokine. Data are given as means \pm SEMs $(n=5-12) .{ }^{*} P<0.01 \mathrm{vs}$. Dp-alone group

group. In addition, although concentration of IL-21 in the supernatants of splenocytes was significantly lower in the $\mathrm{Dp}+\mathrm{nSP} 30$ group than in the Dp-alone group, none of the other measured cytokines (IL-5, 10, 13, and 17) differed between these groups (Additional file 3). Therefore, nSP30 might have affected IgG production without altering systemic Th1 and Th2 immune responses. Because IL21 is a critical factor in IgG production [24, 25], additional study of IL-21 likely would help to clarify the mechanism underlying the reductions in both the IgG1 and IgG2a subtypes.
Recently, the skin was revealed to be the key initial site of allergic sensitization not only for allergic eczema but also for other atopic allergies, including allergic rhinitis, asthma, and food allergies [26-28]. Therefore, skin might play a special role in allergic sensitization. To this end, we assessed the effects of exposure to $\mathrm{Dp}+\mathrm{nSP} 30$ by various other exposure routes on the IgG response (Additional file 4). Consistent with our previously reported results [29], nSP30-mediated IgG suppression was not observed after intranasal, oral, or intradermal administration of $\mathrm{Dp}+\mathrm{nSP} 30$, thus suggesting that the low IgG response 
was a specific effect induced by cutaneous exposure to $\mathrm{Dp}+\mathrm{nSP} 30$.

\section{Effect of cutaneous exposure to Dp + nSP30 on susceptibility to anaphylaxis}

Chemical mediators such as histamine and various cytokines induce the typical symptoms of atopic allergy, including inflammation and itching [30,31]. The production of these chemical mediators is induced when an allergen cross-links IgE molecules bound to highaffinity IgE receptors (FceRI) on mast cells and basophils. However, allergen-specific IgG, considered to be the 'blocking antibody', may neutralize allergen molecules before they can interact with IgE $[32,33]$. Furthermore, IgG-allergen complexes act through a pathway regulated by the inhibitory receptor FcyRIIB to inhibit IgE-mediated mast cell and basophil signaling [32-34]. Therefore, the presence of allergen-specific IgG inhibits IgE-mediated allergic responses. Because our results showed that $\mathrm{Dp}+$ nSP30 led to low levels of Dp-specific IgG with little change in the levels of Dp-specific IgE (Fig. $3 a-c)$, we examined whether this IgE-biased immune response induced by cutaneous exposure to Dp $+\mathrm{nSP} 30$ caused IgE-mediated hypersensitivity to $\mathrm{Dp}$ in a systemic anaphylaxis model. The decrease in rectal temperature after challenge with intravenous $\mathrm{Dp}$ was significantly greater in mice sensitized by $\mathrm{Dp}+\mathrm{nSP} 30$ than in those sensitized by Dp alone (Fig. 4). That is, mice in the Dp + nSP30 group were more sensitive to the induction of Dpspecific anaphylaxis than were those in the Dp-alone group. Together, concurrent cutaneous exposure to $\mathrm{Dp}$ and nSP30 induced IgE-biased immune responses and, subsequently, increased sensitivity to anaphylaxis. In contrast, the functions of specific immunoglobulin subtypes vary between mice and humans; for example, blocking antibodies in humans are considered to be of the IgG4 subtype, which mice lack [35]. Therefore, our results cannot be extrapolated directly to humans. In addition, regulatory $\mathrm{T}$ and $\mathrm{B}$ cells have recently been suggested to be the main suppressors of atopic allergy in allergen-specific immunotherapy [36]. Additional study is needed to clarify whether increases in anaphylactic sensitivity is solely due to blocking antibody.

\section{Effects of sequential cutaneous exposure to allergen and nSP30 on the IgE-biased immune response}

We also evaluated the effects of sequential (rather than concurrent) cutaneous exposure to Dp and nSP30; that is, we topically applied Dp and nSP30 on alternate days rather than applying them together on the same day. Ear thickening and changes in total IgE concentration resulting from alternate-day application of $\mathrm{Dp}$ and nSP30 $(\mathrm{Dp} / \mathrm{nSP} 30)$ did not differ significantly from those resulting from concurrent treatment with Dp and PBS every

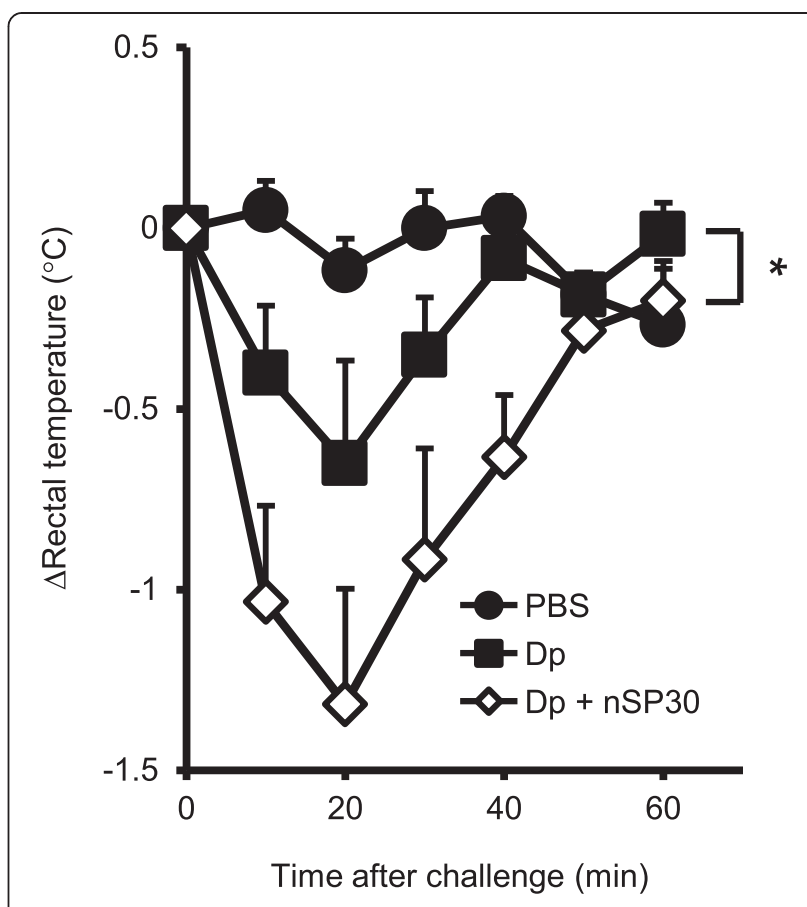

Fig. 4 Sensitivity to anaphylactic shock. Time-dependence of change in rectal temperature in NC/Nga mice due to intravenous challenge with Dp after treatment with Dp alone or Dp $+n S P 30$ in PBS. Data are given as means \pm SEMs $(n=6)$. ${ }^{*} P<0.05$ vs. Dp-alone group

other day (Dp/PBS) (Fig. 5a and b). Taken together, our results indicate that cutaneous exposure to nSP30 did not aggravate Dp-induced AD-like skin lesions regardless of whether the nanoparticles were applied together with or separately from Dp. In addition, alternate-day skin painting with $\mathrm{Dp}$ and nSP30 induced Dp-specific IgE and IgG production and Dp-specific IFN- $\gamma$ - and IL4-secreting splenocytes in the same way as did topical application of Dp and PBS (Fig. 5c-f). Therefore, exposure to both Dp and nSP30 was necessary to induce an IgE-biased immune response. Given that $\mathrm{Dp}+\mathrm{nSP} 30$ formed agglomerates (Fig. 1), an interaction between Dp and nSP30 may have contributed to the subsequent IgEbiased immune response.

\section{Effects of agglomeration of allergen and nSP30 on the IgE-biased immune response}

Because the surface properties of nanomaterials strongly influence their interactions with proteins [37], we investigated how surface modification of nSP30 with carboxyl groups affected the interaction between Dp and nSP30 and the subsequent IgE-biased immune response. Solutions of the mixture of Dp and nSP30 modified with surface carboxyl groups (nSP30C) were clear and colorless (Fig. 1a), and a TEM image of Dp + nSP30C was similar to that of nSP30C alone (Fig. 1b). The mean hydrodynamic diameter of $\mathrm{Dp}+\mathrm{nSP} 30 \mathrm{C}$ was $24.8 \mathrm{~nm}$, which 


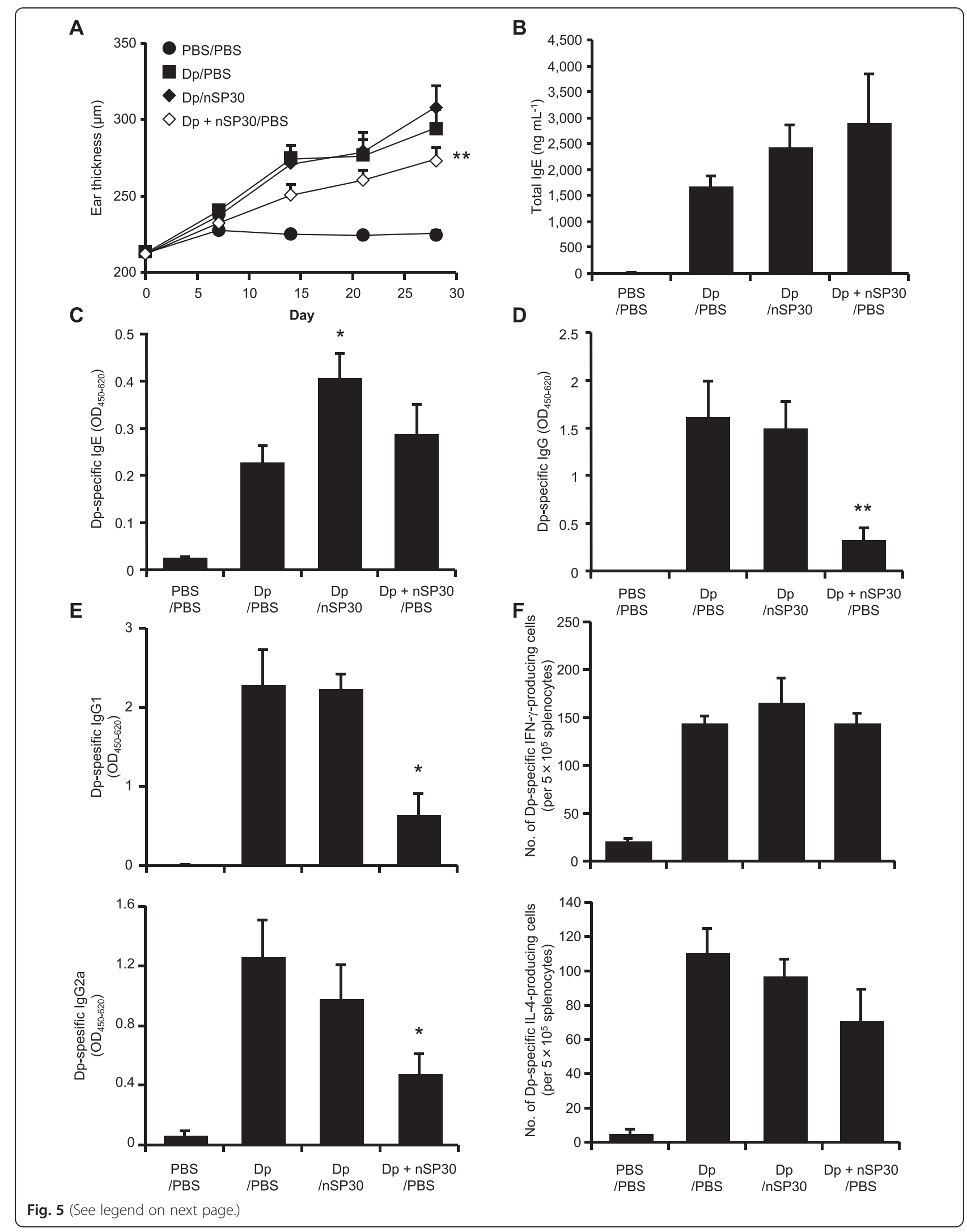


(See figure on previous page.)

Fig. 5 Effects of Dp + nSP30 aggregates compared with Dp and nSP30 administered on alternate days. a Effect of topical application of Dp + nSP30 and PBS (Dp + nSP30/PBS) compared with Dp and nSP30 (Dp/nSP30) on alternate days on ear thickness in NC/Nga mice. b-e Plasma levels of (b) total

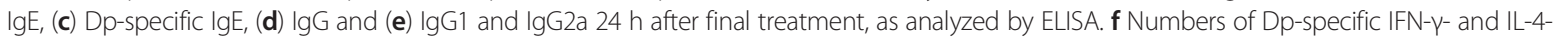
producing splenocytes after re-stimulation with $100 \mu \mathrm{g} \mathrm{mL} \mathrm{m}^{-1} \mathrm{Dp}$, as determined by ELISPOT assays specific for each cytokine. Data are presented as means \pm SEMs $(n=5) .{ }^{*} P<0.05,{ }^{*} P<0.01$ vs. Dp/PBS group

was only approximately $4 \mathrm{~nm}$ larger than that of nSP30C alone (Fig. 1c and d). These results suggest that the mixing of Dp and nSP30C did not result in substantial agglomeration. The level of Dp-specific IgE induced by cutaneous exposure to $\mathrm{Dp}+\mathrm{nSP} 30 \mathrm{C}$ was the same as that induced by cutaneous exposure to $\mathrm{Dp}$ alone or to $\mathrm{Dp}+\mathrm{nSP} 30$ (Fig. 6a). Although cutaneous exposure to Dp + nSP30 significantly reduced the levels of Dp-specific IgG and its subtypes, cutaneous exposure to Dp + nSP30C had little effect on the levels of Dp-induced IgG, IgG1, and IgG2a (Fig. 6b and c).

The vehicle in which nanoparticles are suspended is a key determinant of their dispersion and affects their toxicity $[38,39]$. To further evaluate the effect of agglomeration of $\mathrm{Dp}$ and nSP30 on the IgE-biased immune response, we tested the effect of the vehicle on the formation of agglomerates and the IgE-biased immune response. Solutions of Dp and nSP30, either individually or together, in water were clear and colorless, but the combination of Dp and nSP30 in PBS was cloudy (Fig. 1a). The mean hydrodynamic diameter of nSP30 in water was $19.5 \mathrm{~nm}$ and that of $\mathrm{Dp}+$ nSP30 in water was $20.0 \mathrm{~nm}$ (Fig. 1c and d), indicating that mixing Dp and nSP30 in water did not cause agglomeration. We repeatedly painted NC/Nga mice with $\mathrm{Dp}+$ nSP30 in water and evaluated the Dp-induced antibody responses. When suspended in water, nSP30 had no effect on the level of Dp-specific IgE (Fig. 7a). In addition, the levels of Dp-specific total IgG, IgG1, and IgG2a did not differ between the $\mathrm{Dp}+\mathrm{nSP} 30$ and $\mathrm{Dp}$-alone groups when the vehicle used was water (Fig. $7 b$ and $c$ ). Therefore

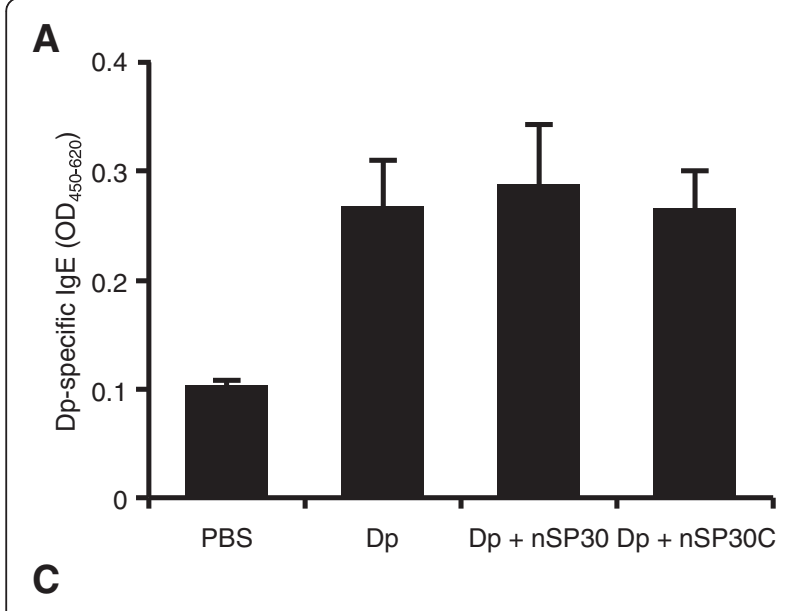

B
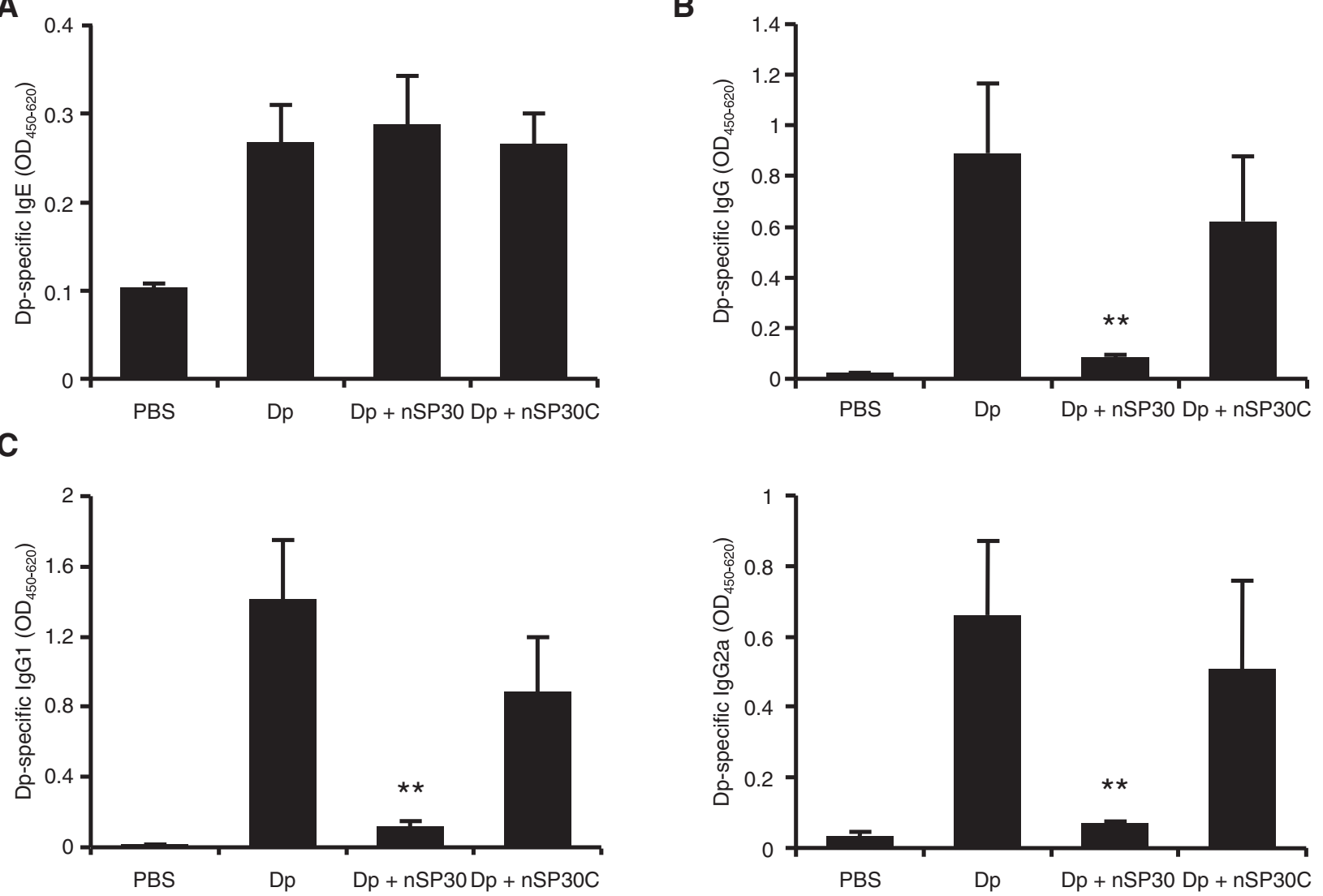

Fig. 6 Prevention of Dp + nSP30-mediated IgE-biased immune response by surface modification of nSP30. a-c Plasma levels of Dp-specific (a) $\lg E_{1}(\mathbf{b}) \lg G$, and (c) $\lg G 1$ and $\lg G 2 \mathrm{a}$ at $24 \mathrm{~h}$ after final treatment of NC/Nga mice with Dp alone, Dp + nSP30, or Dp + nSP30C, as analyzed by ELISA. Data are given as means \pm SEMs $(n=5) .{ }^{*} P<0.05,{ }^{*} P<0.01$ vs. Dp-alone group 


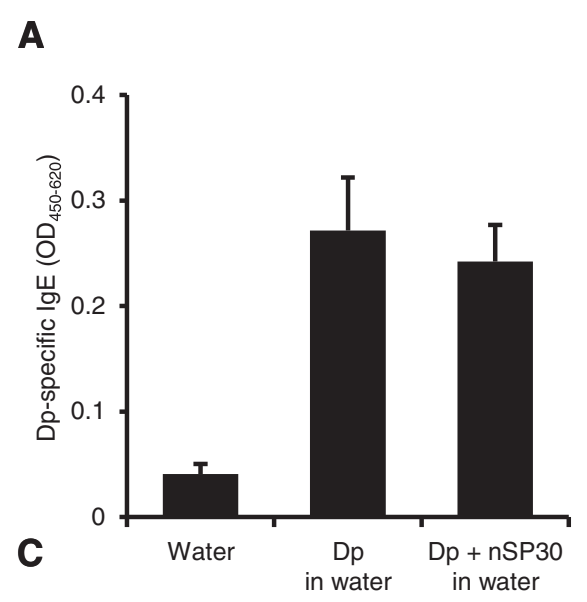

B
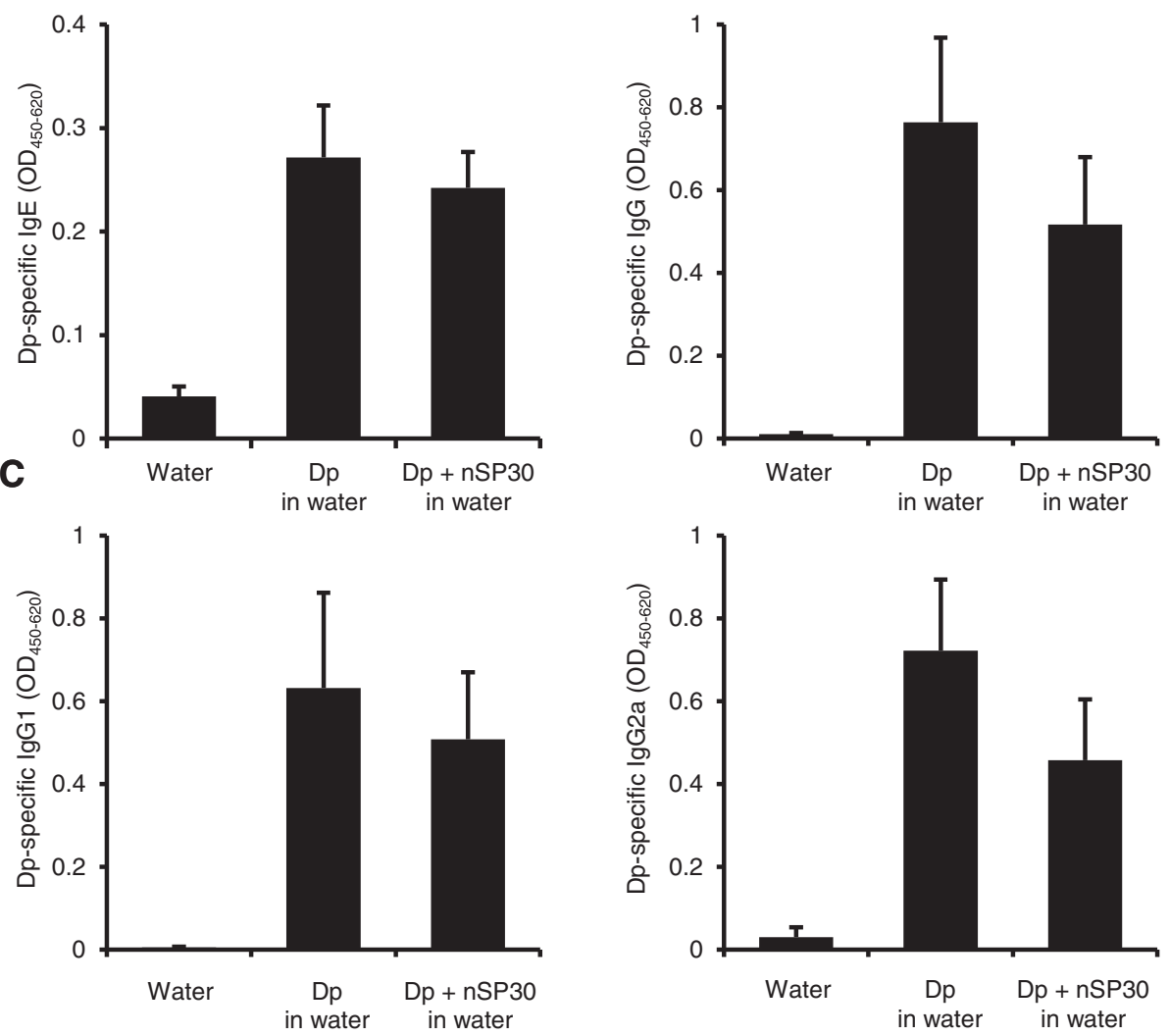

Fig. 7 Effect of topical application of dispersed mixture of Dp + nSP30 on antibody response. a-c Plasma levels of Dp-specific (a) IgE, (b) IgG, and (c) IgG1 and IgG2a at $24 \mathrm{~h}$ after final topical treatment of NC/Nga mice with Dp alone or Dp + nSP30 in water, as analyzed by ELISA. Data are given as means \pm SEMs ( $n=5$ or 6 ). ${ }^{*} P<0.05,{ }^{* *} P<0.01$ vs. Dp-alone group

the agglomeration of Dp and nSP30 might be necessary to impede the allergen-specific IgG response, but it did not affect the IgE response.

Atopic patients typically have relatively low IgG/IgE ratios $[40,41]$. In addition, human studies indicate that IgE production in the absence of IgG production is a key risk factor for the onset of atopic allergy [42]. Low-dose exposure to allergen sometimes induces a low IgG/IgE ratio in both mice and humans $[43,44]$. Therefore, the formation of agglomerates in our experimental system may, in effect, decrease allergen doses and then induce an IgE-biased immune response. To assess the amount of unbound Dp, we compared the Dp concentration in the supernatant after centrifugation. After centrifugation, the Dp concentration in the supernatant of the Dp-alone group was somewhat lower than that observed before centrifugation $(1000 \mu \mathrm{g} / \mathrm{mL} \rightarrow 457 \mu \mathrm{g} / \mathrm{mL}$ ) (Fig. 8). Mixing $\mathrm{Dp}$ and nSP30 together in PBS decreased the Dp concentrations in the supernatants in an nSP30dependent manner (Fig. 8). In contrast, mixing Dp with nSP30C in PBS or with nSP30 in water had little effect on the Dp concentration in the supernatant compared with that of the Dp-alone group. Therefore, agglomeration might increase the amount of $\mathrm{Dp}$ absorbed to nSP30. Considering the fact that, in general, aggregates and agglomerates of nanomaterials have difficulty penetrating the skin barrier [45], the agglomeration of Dp and nSP30 might decrease the exposure dose of Dp somewhat even when the agglomerates release Dp on the skin.

We previously examined the $\mathrm{Dp}$ dose-effect in the same NC/Nga model (Hirai T. et al., submitted paper). In that study, levels of both Dp-specific IgE and IgG were correlated with exposure amount of $\mathrm{Dp}$ in the range of 30 to $120 \mu \mathrm{g} \mathrm{Dp}$ mouse $^{-1}$; in the current study, we used a dose of $120 \mu \mathrm{g}$ Dp mouse ${ }^{-1}$ for the Dp-alone group. Furthermore, the Th2-related cytokine response in Dp-stimulated splenocytes was lower in the120- $\mu$ g Dp mouse $^{-1}$ group than in other (lower) dose groups. In contrast, we show here that the exposure to agglomerates of Dp and nSP30 in PBS induced a dampened Dp-specific IgG response with little change in the Dp-specific IgE and Th1/Th2 immune responses compared with those of the Dp-alone group (Fig. 3 and Additional file 3). Together, 

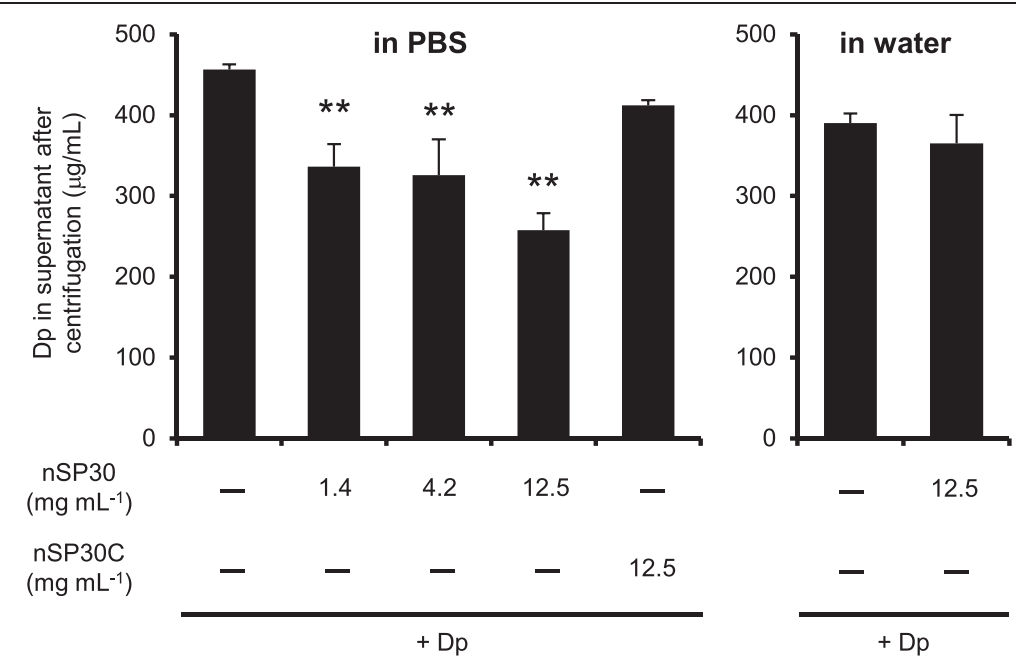

Fig. 8 Evaluation of absorption of Dp to nSP30. The amount of Dp in the supernatant of each centrifuged sample was determined $\left(\mathrm{OD}_{280}\right)$. Data are given as means $\pm \mathrm{SD}(n=3) .{ }^{*} P<0.01 \mathrm{vs}$. Dp-alone

these observations suggest that the IgE-biased immune response in the current study was not solely due to the low Dp exposure resulting from the agglomeration of Dp and nSP30. Perhaps the agglomerates of Dp and nSP30 in PBS not only decreased the Dp exposure dose but also behaved as a 'depot,' thus controlling the allergen concentration, prolonging allergen exposure, and subsequently causing IgE-biased allergic sensitization. We believe additional studies that focus on the effect of nanoparticles on allergen penetration kinetics in the skin would be beneficial to confirm the safety of cutaneous exposure to nanomaterials. However, we acknowledge the need for additional studies in which the exposure scenario is more representative of that in humans to define the safety of concurrent cutaneous exposure to nanomaterials and allergen.

It is well recognized that there are some situations in our daily lives, the development of atopic allergy is inhibited by higher dose exposure to allergen due to high induction of blocking IgG [46-48]. Particularly in these high-dose exposure situations, we suggest that allergen-nanoparticle agglomerates, by inducing IgEbiased immune responses, might play a critical role in the development of atopic allergy. In addition, the IgEbiased immune response induced by cutaneous exposure to agglomerates of allergen and nanoparticles in our mice was similar to those humans with atopic allergies, who often have a low IgG/IgE ratio, as mentioned earlier $[40,41]$. Therefore epidemiologic studies that address cutaneous as well as inhalational exposure to nanomaterials may improve our understanding of the onset of atopic allergy.

Recently, Ilves M. et al. described the effects of cutaneous exposure to nano-sized $\mathrm{ZnO}(\mathrm{nZnO})$ administered with model antigens, ovalbumin and staphylococcal enterotoxin $\mathrm{B}$, on AD-like skin lesions and antibody responses [49]. Interestingly, the effects observed for $\mathrm{nZnO}$ and an antigen were similar to the effects of agglomerates of $\mathrm{Dp}$ and nSP30: $\mathrm{nZnO}$ suppressed allergen-induced skin inflammation and induced low-level IgG production in the context of a high IgE response. The authors of the previous study [49] did not address changes of nZnO dispersibility by mixing allergen, but considering that $\mathrm{nZnO}$ is predisposed to forming agglomerates and might adsorb a coexisting substance [50], nZnO might play similar role to that of nSP30. To better understand the hazards of nanomaterials so that we can maximize their potential benefits, we should pay increased attention to the state of nanoparticles (e.g. dispersibility) in administration in nanotoxicology studies. We consider this focus particularly applicable in the hazard evaluation of nanomaterials that are in the presence of other substances, which could interact with them.

Cutaneous exposure to aggregates or agglomerates of nanomaterials is generally considered to be safer than is similar exposure to individual nanoparticles, mainly because agglomerates of nanomaterials have difficulty penetrating the skin [50]. However, Dp and nSP30 induced an IgE-biased immune response only when they formed agglomerates. Although these results represent only indirect effects of nanomaterials, we think that hazard identification is necessary even when nanomaterials are considered to be unable to cross the skin barrier (e.g. when nanomaterials form aggregates or agglomerates). In contrast, surface modification of the nSP30 with carboxyl groups suppressed the adsorption of the allergen and did not induce IgE-biased allergic sensitization (Fig. 6). An increased understanding of the regulatory factors that 
induce the agglomeration of silica nanoparticles is crucial for appropriate regulation of the surface properties of nanomaterials so that they can be used safely.

\section{Conclusions}

Cutaneous exposure to agglomerates of $\mathrm{Dp}$ and nSP30 induced an IgE-biased immune response in NC/Nga mice and increased their sensitivity to anaphylaxis. Surprisingly, these results were independent of the innate biologic effects of nSP30; these results required both simultaneous exposure to Dp and nSP30 and their agglomeration. In particular, features of the mice with IgEbiased immune response induced by cutaneous exposure to agglomerates of allergen and nanoparticles resembled those of humans with atopic allergies, who often have a low IgG/IgE ratio; follow-up epidemiologic studies that focus cutaneous compared with inhalational exposure to nanomaterials might improve our understanding of the onset of atopic allergy. In light of our findings, we suggest that minimizing the interaction between nanomaterials and allergens may improve the safety of topically applied products containing nanomaterials.

\section{Methods}

\section{Silica nanoparticles}

nSP30 and nSP30C (nSP30C is a surface modified nSP30 with carboxyl groups) silica nanoparticles (diameter, $30 \mathrm{~mm}$ ) were purchased from Micromod Partikeltechnologie (Rostock/Warnemünde, Germany). Suspensions of silica nanoparticles were stored at room temperature. Immediately prior to use, the suspensions were sonicated at $400 \mathrm{~W}$ for $5 \mathrm{~min}$ at $25^{\circ} \mathrm{C}$ and then vortexed for $1 \mathrm{~min}$.

\section{Mice}

Female NC/Nga slc mice were purchased from SLC (Kyoto, Japan) and used at 6 wk of age. All animal experiments were performed in accordance with the institutional guidelines of Osaka University and National Institute of Biomedical Innovation regarding the ethical treatment of animals.

\section{Preparation of the mixtures of Dp and nSP30s}

To prepare the mixtures of nSP30s and Dp (Cosmo Bio LSL, Tokyo, Japan), we first combined nSP30s ( $25 \mathrm{mg} \mathrm{mL}^{-1}$ in water) and concentrated PBS $(1.1 \times$ to $4.6 \times$; the concentration of the PBS stock used depended on the concentration of nSP30s needed in the final sample, the diluent for which was $1 \times$ PBS). We then added the stock solution of $\mathrm{Dp}$ (2.78 $\mathrm{mg}$ protein $\mathrm{mL}^{-1}$ in PBS) to the solutions containing various concentrations of nSP30s. As soon as the nSP30s and Dp solutions were combined, the mixtures were vortexed for $1 \mathrm{~min}$, allowed to incubate at room temperature for 0.5 to $1.5 \mathrm{~h}$, and vortexed again for $1 \mathrm{~min}$ just prior to use.

\section{Physicochemical examination of silica nanoparticles}

TEM (H-7650; Hitachi High-Technologies Corporation, Tokyo, Japan) was used to assess the size and shape of the silica nanoparticles. nSP30 and nSP30C nanoparticles were diluted to $0.25 \mathrm{mg} \mathrm{mL}^{-1}$ in PBS or deionized water, and the mean particle diameter and zeta potential at $25{ }^{\circ} \mathrm{C}$ were measured by using a Zetasizer Nano ZS (Malvern Instruments, Worcestershire, UK). Specifically, the mean diameters and particle size distributions of the nanoparticles were measured by means of a dynamic light-scattering method, whereas zeta potentials were measured by laser Doppler electrophoresis; both types of measurements were performed by using capillary cells (Malvern Instruments). The $\mathrm{pH}$ of each particle suspension was measured by using an ISFET $\mathrm{pH}$ meter (Shindengen, Tokyo, Japan).

\section{Skin painting and assessment of allergic response}

Using a hair-removal cream (Epilat; Kracie, Tokyo, Japan), we removed the hair from the backs of all mice twice during each experiment (on day 1 and on day 18-20). Mice were treated either with $\mathrm{Dp}\left(1 \mathrm{mg} \mathrm{mL}^{-1}\right.$ fin. conc) alone or with silica nanoparticles (nSP30 or nSP30C, $12.5 \mathrm{mg} \mathrm{mL}^{-1}$ fin. conc.) in $120 \mu \mathrm{L}$ PBS by painting the ventral side of both ears and the depiliated dorsum (20 $\mu \mathrm{L}$ per ear and $80 \mu \mathrm{L}$ on the back) on alternate days or every third day for $4 \mathrm{wk}$ for a total of 13 applications. Additional mice were painted with $\mathrm{Dp}$ only on one day followed by painting with nSP30 alone on the next day; this alternating pattern was repeated for a total of 13 applications of each solution over 4 wk. Regardless of the dose schedule used, any sample remaining from a previous application was removed by gently wiping with a disposable wipe (Kim wipes, Crecia, Tokyo, Japan) wetted with $70 \%$ ethanol.

We used a dial thickness gauge (0.001-mm type; Ozaki Manufacturing, Tokyo, Japan) to measure ear thickness weekly. To evaluate the severity of the IgE-mediated allergic response' instead, we used a systemic anaphylaxis model. Specifically, at $1 \mathrm{wk}$ after the final skin painting, each mouse was challenged with an intravenous injection of Dp (15 $\mu$ g in $200 \mu \mathrm{L}$ PBS). The severity of anaphylactic shock was assessed according to the change in rectal temperature measured by using a digital thermometer (KN-91; Natsume Seisakusho, Tokyo, Japan).

\section{Histologic analysis}

At $24 \mathrm{~h}$ after the final topical treatment, the ears of euthanized mice were removed, placed in fixative solution (4\% paraformaldehyde in PBS; Wako, Osaka, Japan), embedded in paraffin, and sectioned. The tissue sections were stained with hematoxylin and eosin or with toluidine blue. Histopathological examination was performed by the Applied Medical Research Laboratory (Osaka, 
Japan). For each sample, representative symptoms of AD (scab formation, acanthosis and inflammatory cell infiltration) were scored as follows: 0 , none; 1 , slight; 2 , mild; 3 , moderate; and 4, severe. In addition, we counted the number of mast cells in 3 random high-power fields (magnification, 400x).

\section{Blood sampling}

Using hematocrit capillary tubes (Terumo, Tokyo, Japan), we obtained blood samples from the retro-orbital venous plexus once each week during the study period. At $24 \mathrm{~h}$ after the final skin painting, blood was collected by cardiocentesis into heparin-treated syringes and centrifuged at $3000 \times g$ at $4{ }^{\circ} \mathrm{C}$; the resulting plasma was stored at $-80{ }^{\circ} \mathrm{C}$ until analysis.

\section{Quantitation of total IgE concentration}

The total IgE concentration in plasma was measured by using an ELISA kit (BD Biosciences, San Diego, CA, USA) according to the manufacturer's instructions.

\section{Detection of Dp-specific antibody}

The levels of Dp-specific antibody in plasma were determined by ELISA. To detect IgG, IgG1, IgG2a, IgG2b and IgG3, we coated ELISA plates (Maxisorp, type 96 F; Nunc A/S, Roskilde, Denmark) with Dp in PBS $\left(50 \mu \mathrm{g} \mathrm{mL}{ }^{-1}\right)$. The coated plates were incubated with $2 \%$ Block Ace (Dainippon Sumitomo Pharmaceuticals, Osaka, Japan). Plasma samples were diluted by $2 \%$ Block Ace and these dilutions were added to the Dp-coated plates. After incubation with plasma, the coated plates were incubated with a horseradish peroxidase-conjugated goat anti-mouse IgG, IgG1, IgG2a, IgG2b or IgG3 solution (SouthernBiotech, Birmingham, AL, USA) for two hours at room temperature. After the incubation, the color reaction was developed with tetramethyl benzidine (Moss, Inc.; Pasadena, MD, USA), stopped with $2 \mathrm{~N} \mathrm{H}_{2} \mathrm{SO}_{4}$, and measured at $\mathrm{OD}_{450-620}$ on a microplate reader.

To detect Dp-specific IgE, we coated ELISA plates (Maxisorp, type $96 \mathrm{~F}$ ) with purified rat anti-mouse IgE (2 $\mu \mathrm{g} \mathrm{mL}^{-1}$; R35-72, BD Biosciences;), incubated them with Block Ace, and added samples of diluted plasma as described earlier. Treated plates were incubated with biotin-conjugated Dp $\left(5 \mu \mathrm{g} \mathrm{mL}^{-1}\right)$ followed by horseradish peroxidase-coupled streptavidin (Southern Biotechnology Associates). Color detection was performed as described earlier.

\section{Isolation of splenocytes}

Spleens were removed aseptically and placed in RPMI 1640 (Wako) supplemented with $10 \%$ fetal bovine serum, $10 \mathrm{~mL} \mathrm{~L}^{-1}$ of a $100 \times$ nonessential amino acid solution (Gibco, Invitrogen, Carlsbad, CA, USA), $50 \mu \mathrm{M}$ 2-mercaptoethanol (Gibco), and $1 \%$ antibiotic cocktail $\left(10,000 \mathrm{U} \mathrm{mL}^{-1}\right.$ penicillin, $10,000 \mu \mathrm{g} \mathrm{mL} \mathrm{L}^{-1}$ streptomycin, $25 \mu \mathrm{g} \mathrm{mL} \mathrm{m}^{-1}$ amphotericin B; Gibco). Single-cell suspensions of splenocytes were treated with ammonium chloride to lyse the red blood cells; treated splenocytes then were washed, counted, and suspended in RPMI 1640.

\section{Cytokine assays}

To determine antigen-specific cytokine (IFN- $\gamma$ and IL-4) responses, splenocytes $\left(5 \times 10^{5}\right.$ cells well $\left.^{-1}\right)$ were stimulated with Dp antigen $\left(100 \mu \mathrm{g} \mathrm{mL}{ }^{-1}\right)$ in vitro. After $24 \mathrm{~h}$ incubation at $37{ }^{\circ} \mathrm{C}(95 \%$ room air, $5 \% \mathrm{CO} 2)$, stimulated splenocytes were washed, and the numbers of IFN$\gamma$ - and IL-4-producing cells were determined by using an ELISPOT assay kit (BD Biosciences) according to the manufacturer's instructions.

\section{Measurement of amount of $\mathrm{Dp}$ adsorbed to silica nanoparticles}

Solutions of Dp (1 mg mL ${ }^{-1}$ fin. conc.) + each nSP immediately after preparation in $\mathrm{PBS}$ or water for $30 \mathrm{~min}$ at $25{ }^{\circ} \mathrm{C}$ were centrifuged $\left(2 \mathrm{~h}, 40,000 \times g, 4{ }^{\circ} \mathrm{C}\right)$. The amount of $\mathrm{Dp}$ in the supernatant then was determined spectrophotometrically $\left(\mathrm{OD}_{280}\right.$; Nanodrop 2000 , Thermo Scientific, Kanagawa, Japan).

\section{Statistical analysis}

Statistical analyses were performed with Ekuseru-Toukei 2010 software (Social Survey Research Information Co., Ltd., Tokyo, Japan). All data are presented as means \pm SEMs. Significant differences between control groups and experimental groups were determined by using the Dunnett test; a $P$ value less than 0.05 was considered significant.

Methods for additional files are available in (Additional file 5).

\section{Additional files}

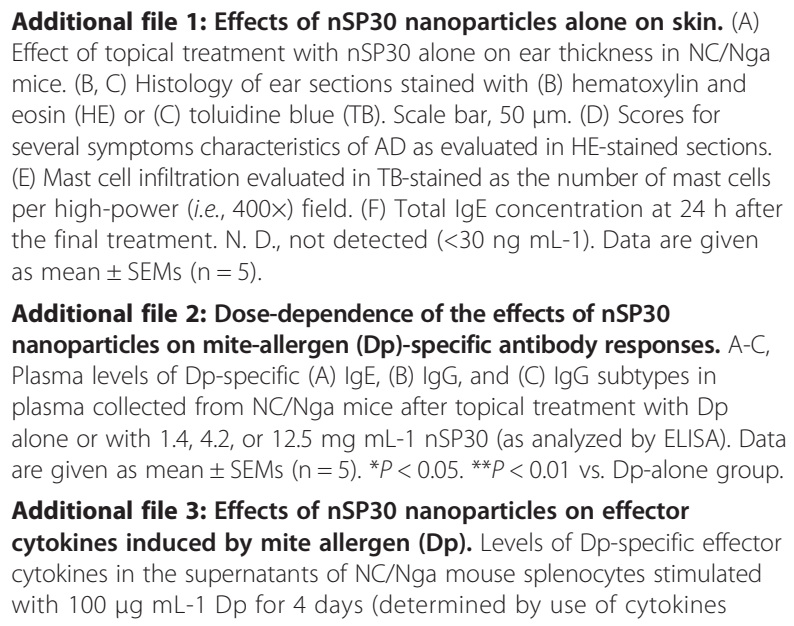

Additional file 2: Dose-dependence of the effects of nSP30 nanoparticles on mite-allergen (Dp)-specific antibody responses. $A-C$, Plasma levels of Dp-specific (A) lgE, (B) lgG, and (C) lgG subtypes in plasma collected from NC/Nga mice after topical treatment with Dp alone or with $1.4,4.2$, or $12.5 \mathrm{mg} \mathrm{mL}-1 \mathrm{nSP} 30$ (as analyzed by ELISA). Data are given as mean \pm SEMs $(n=5)$. ${ }^{*} P<0.05 .{ }^{*} P<0.01 \mathrm{vs}$. Dp-alone group.

Additional file 3: Effects of nSP30 nanoparticles on effector cytokines induced by mite allergen (Dp). Levels of Dp-specific effector cytokines in the supernatants of NC/Nga mouse splenocytes stimulated with $100 \mu \mathrm{g} \mathrm{mL-1}$ Dp for 4 days (determined by use of cytokines 
immunoassay or ELISA). Data are given as means $\pm \operatorname{SEMS}(n=5) .{ }^{*} P<$ 0.05. ${ }^{*} P<0.01$ vs. Dp-alone group.

Additional file 4: Effects of administration route of mite allergen (Dp) + nSP30 mamoparticles on Dp-sepcific antibody response. Levels of Dp-specific IgE and IgG in plasma collected from NC/Nga mice after treatment with Dp alone or Dp + nSP30 by intradermal (foot-pad injection), intranasal, or oral administration (as analyzed by ELISA). Data are given as means \pm SEMs $(n=3-6)$. ${ }^{*} P<0.01$ vs. Dp-alone group.

\section{Additional file 5: Additional methods.}

\section{Competing interests}

The authors declare that they have no competing interests.

\section{Authors' contributions}

TH and YY designed the study; TH, HT, Kl, AU, TM, and NN performed experiments; $T H$ and $Y Y$ collected and analyzed data; $T H$ and $Y Y$ wrote the manuscript; TYoshida, KN, HK, ST, TT, IKJ, HN, TYoshikawa, and KH provided technical support and conceptual advice. YT supervised all the projects. All authors discussed the results and commented on the manuscript. All authors read and approved the final mansucript.

\section{Acknowledgements}

This study was supported by Grants-in-Aid for Scientific Research from the Ministry of Education, Culture, Sports, Science and Technology of Japan (MEXT) and from the Japan Society for the Promotion of Science (JSPS); by a Grant-in-Aid for JSPS Fellows; by Health Labour Sciences Research Grants from the Ministry of Health, Labour and Welfare of Japan (MHLW); by The Takeda Science Foundation; by The Research Foundation for Pharmaceutical Sciences; by The Japan Food Chemical Research Foundation; by the Urakami Foundation; and by the Uehara Memorial Foundation.

\section{Author details}

'Laboratory of Toxicology and Safety Science, Graduate School of Pharmaceutical Sciences, Osaka University, 1-6 Yamadaoka, Suita, Osaka 565-0871, Japan. ${ }^{2}$ Vaccine Creation Project, BIKEN Innovative Vaccine Research Alliance Laboratories, Research Institute for Microbial Diseases, Osaka University, 3-1 Yamadaoka, Suita, Osaka 565-0871, Japan. 'BIKEN Center for Innovative Vaccine Research and Development, The Research Foundation for Microbial Diseases of Osaka University, 3-1 Yamadaoka, Suita, Osaka 565-0871, Japan. ${ }^{4}$ Laboratory of Innovative Antibody Engineering and Design, Center for Drug Innovation and Screening, National Institute of Biomedical Innovation, 7-6-8 Saitoasagi, Ibaraki, Osaka 567-0085, Japan. ${ }^{5}$ Laboratory of Biopharmaceutical Research, National Institute of Biomedical Innovation, 7-6-8 Saitoasagi, Ibaraki, Osaka 567-0085, Japan. ${ }^{6}$ The Center for Advanced Medical Engineering and Informatics, Osaka University, 1-6 Yamadaoka, Suita, Osaka 565-0871, Japan. 'Laboratory of Environmental Pharmacometrics, Graduate School of Pharmaceutical Sciences, Osaka University, 1-6 Yamadaoka, Suita, Osaka 565-0871, Japan. ${ }^{8}$ Genome Information Research Center, Research Institute for Microbial Diseases, Osaka University, 3-1 Yamadaoka, Suita, Osaka 565-0871, Japan. ${ }^{9}$ Laboratory of Adjuvant Innovation, National Institute of Biomedical Innovation, 7-6-8 Saitoasagi, Ibaraki, Osaka 567-0085, Japan. ${ }^{10}$ Laboratory of Vaccine Science, Immunology Frontier Research Center, World Premier International Research Center, Osaka University, 3-1 Suita, Osaka 565-0871, Japan. ${ }^{11}$ Division of Foods, National Institute of Health Sciences, 1-18-1 Kamiyoga, Setagaya-ku, Tokyo 158-8501, Japan.

Received: 18 April 2015 Accepted: 16 June 2015 Published online: 26 June 2015

\section{References}

1. Auffan M, Rose J, Bottero JY, Lowry GV, Jolivet JP, Wiesner MR. Towards a definition of inorganic nanoparticles from an environmental, health and safety perspective. Nat Nanotechnol. 2009;4(10):634-41. doi:10.1038/nnano.2009.242.

2. Cheng Z, Al Zaki A, Hui JZ, Muzykantov VR, Tsourkas A. Multifunctional nanoparticles: cost versus benefit of adding targeting and imaging capabilities. Science. 2012;338(6109):903-10. doi:10.1126/science.1226338.

3. Bowman DM, van Calster G, Friedrichs S. Nanomaterials and regulation of cosmetics. Nat Nanotechnol. 2010;5(2):92. doi:10.1038/nnano.2010.12.
4. Jain RK, Stylianopoulos T. Delivering nanomedicine to solid tumors. Nat Rev Clin Oncol. 2010;7(11):653-64. doi:10.1038/nrclinonc.2010.139.

5. Peters R, Kramer E, Oomen AG, Rivera ZE, Oegema G, Tromp PC, et al. Presence of nano-sized silica during in vitro digestion of foods containing silica as a food additive. ACS Nano. 2012;6(3):2441-51. doi:10.1021/nn204728k

6. Yamashita K, Yoshioka Y, Higashisaka K, Mimura K, Morishita Y, Nozaki M, et al. Silica and titanium dioxide nanoparticles cause pregnancy complications in mice. Nat Nanotechnol. 2011;6(5):321-8. doi:10.1038/nnano.2011.41.

7. Setyawati MI, Tay CY, Chia SL, Goh SL, Fang W, Neo MJ, et al. Titanium dioxide nanomaterials cause endothelial cell leakiness by disrupting the homophilic interaction of VE-cadherin. Nat Commun. 2013;4:1673. doi:10.1038/ncomms2655.

8. Kulbok PA, Baldwin JH. From preventive health behavior to health promotion: advancing a positive construct of health. ANS Adv Nurs Sci. 1992;14(4):50-64.

9. Janssen NA, Brunekreef B, van Vliet P, Aarts F, Meliefste K, Harssema H, et al. The relationship between air pollution from heavy traffic and allergic sensitization, bronchial hyperresponsiveness, and respiratory symptoms in Dutch schoolchildren. Environ Health Perspect. 2003;111(12):1512-8.

10. Bartra J, Mullol J, del Cuvillo A, Davila I, Ferrer M, Jauregui I, et al. Air pollution and allergens. J Investig Allergol Clin Immunol. 2007;17 Suppl 2:3-8.

11. McCreanor J, Cullinan P, Nieuwenhuijsen MJ, Stewart-Evans J, Malliarou E, Jarup L, et al. Respiratory effects of exposure to diesel traffic in persons with asthma. N Engl J Med. 2007;357(23):2348-58.

12. Tsuji JS, Maynard AD, Howard PC, James JT, Lam CW, Warheit DB, et al. Research strategies for safety evaluation of nanomaterials, part IV: risk assessment of nanoparticles. Toxicol Sci. 2006;89(1):42-50.

13. Som C, Wick P, Krug H, Nowack B. Environmental and health effects of nanomaterials in nanotextiles and facade coatings. Environ Int. 2011;37(6):1131-42. doi:10.1016/j.envint.2011.02.013.

14. Rancan F, Gao Q, Graf C, Troppens S, Hadam S, Hackbarth S, et al. Skin penetration and cellular uptake of amorphous silica nanoparticles with variable size, surface functionalization, and colloidal stability. ACS Nano. 2012:6(8):6829-42. doi:10.1021/nn301622h.

15. Dhanirama D, Gronow J, Voulvoulis N. Cosmetics as a potential source of environmental contamination in the UK. Environ Technol. 2012;33(13-15):1597-608.

16. Li N, Xia T, Nel AE. The role of oxidative stress in ambient particulate matter-induced lung diseases and its implications in the toxicity of engineered nanoparticles. Free Radic Biol Med. 2008;44(9):1689-99. doi:10.1016/j.freeradbiomed.2008.01.028.

17. Matsuda H, Watanabe N, Geba GP, Sperl J, Tsudzuki M, Hiroi J, et al. Development of atopic dermatitis-like skin lesion with lgE hyperproduction in NC/Nga mice. Int Immunol. 1997;9(3):461-6.

18. Banerjee S, Resch Y, Chen KW, Swoboda I, Focke-Tejkl M, Blatt K, et al. Der p 11 is a major allergen for house dust mite-allergic patients suffering from atopic dermatitis. J Investig Dermatol. 2015;135(1):102-9. doi:10.1038/ jid.2014.271.

19. Fuiano N, Diddi G, Delvecchio M, Cl C. Prevalence of positive atopy patch test in an unselected pediatric population. Clin Mol Allergy. 2015;13(1):2. doi:10.1186/s12948-015-0011-2.

20. Aioi A, Tonogaito H, Suto H, Hamada K, Ra CR, Ogawa H, et al. Impairment of skin barrier function in NC/Nga Tnd mice as a possible model for atopic dermatitis. Br J Dermatol. 2001;144(1):12-8.

21. Flohr C, Johansson SG, Wahlgren CF, Williams H. How atopic is atopic dermatitis? J Allergy Clin Immunol. 2004;114(1):150-8. doi:10.1016/ j.jaci.2004.04.027.

22. Yagi $R$, Nagai $H$, ligo $Y$, Akimoto $T$, Arai T, Kubo M. Development of atopic dermatitis-like skin lesions in STAT6-deficient NC/Nga mice. J Immunol. 2002;168(4):2020-7.

23. Coffman RL, Savelkoul HF, Lebman DA. Cytokine regulation of immunoglobulin isotype switching and expression. Semin Immunol. 1989;1(1):55-63.

24. Ozaki K, Spolski R, Feng CG, Qi CF, Cheng J, Sher A, et al. A critical role for IL-21 in regulating immunoglobulin production. Science. 2002;298(5598):1630-4. doi:10.1126/science.1077002.

25. Vogelzang A, McGuire HM, Yu D, Sprent J, Mackay CR, King C. A fundamental role for interleukin-21 in the generation of $\mathrm{T}$ follicular helper cells. Immunity. 2008;29(1):127-37. doi:10.1016/j.immuni.2008.06.001. 
26. Palmer CN, Irvine AD, Terron-Kwiatkowski A, Zhao Y, Liao H, Lee SP, et al. Common loss-of-function variants of the epidermal barrier protein filaggrin are a major predisposing factor for atopic dermatitis. Nat Genet. 2006;38(4):441-6.

27. Weidinger S, O'Sullivan M, Illig T, Baurecht H, Depner M, Rodriguez E, et al. Filaggrin mutations, atopic eczema, hay fever, and asthma in children. J Allergy Clin Immunol. 2008;121(5):1203-9. doi:10.1016/j.jaci.2008.02.014. 5.

28. Lack G. Update on risk factors for food allergy. J Allergy Clin Immunol. 2012;129(5):1187-97. doi:10.1016/j.jaci.2012.02.036.

29. Hirai T, Yoshikawa T, Nabeshi H, Yoshida T, Tochigi S, Ichihashi K, et al. Amorphous silica nanoparticles size-dependently aggravate atopic dermatitis-like skin lesions following an intradermal injection. Part Fibre Toxicol. 2012;9:3. doi:10.1186/1743-8977-9-3.

30. Kawakami T, Ando T, Kimura M, Wilson BS, Kawakami Y. Mast cells in atopic dermatitis. Curr Opin Immunol. 2009;21(6):666-78. doi:10.1016/ j.coi.2009.09.006

31. Stone KD, Prussin C, Metcalfe DD. IgE, mast cells, basophils, and eosinophils. J Allergy Clin Immunol. 2010;125(2 Suppl 2):S73-80. doi:10.1016/ j.jaci.2009.11.017

32. Strait RT, Morris SC, Finkelman FD. IgG-blocking antibodies inhibit lgE-mediated anaphylaxis in vivo through both antigen interception and Fc gamma Rllb cross-linking. J Clin Invest. 2006;116(3):833-41. doi:10.1172/JCI25575.

33. Uermosi C, Beerli RR, Bauer M, Manolova V, Dietmeier K, Buser RB, et al. Mechanisms of allergen-specific desensitization. J Allergy Clin Immunol. 2010;126(2):375-83. doi:10.1016/j.jaci.2010.05.040.

34. Cassard L, Jonsson F, Arnaud S, Daeron M. Fcgamma receptors inhibit mouse and human basophil activation. J Immunol. 2012;189(6):2995-3006. doi:10.4049/jimmunol.1200968.

35. Jutel $M$, Jaeger $L$, Suck $R$, Meyer $H$, Fiebig $H$, Cromwell $O$. Allergen-specific immunotherapy with recombinant grass pollen allergens. J Allergy Clin Immunol. 2005;116(3):608-13. doi:10.1016/j.jaci.2005.06.004.

36. Akdis M, Akdis CA. Mechanisms of allergen-specific immunotherapy: multiple suppressor factors at work in immune tolerance to allergens. J Allergy Clin Immunol. 2014;133(3):621-31. doi:10.1016/j.jaci.2013.12.1088.

37. Nel AE, Madler L, Velegol D, Xia T, Hoek EM, Somasundaran $P$, et al. Understanding biophysicochemical interactions at the nano-bio interface. Nat Mater. 2009;8(7):543-57. doi:10.1038/nmat2442.

38. Jiang J. Oberdo"rster Gn, Biswas P. Characterization of size, surface charge, and agglomeration state of nanoparticle dispersions for toxicological studies. J Nanoparticle Res. 2009;11(1):77-89.

39. Boverhof DR, David RM. Nanomaterial characterization: considerations and needs for hazard assessment and safety evaluation. Anal Bioanal Chem. 2010;396(3):953-61. doi:10.1007/s00216-009-3103-3.

40. Chapman MD, Platts-Mills TA. Measurement of IgG, IgA and IgE antibodies to Dermatophagoides pteronyssinus by antigen-binding assay, using a partially purified fraction of mite extract (F4P1). Clin Exp Immunol. 1978:34(1):126-36.

41. Platts-Mills TA. Local production of $\lg G, \lg A$ and $\lg E$ antibodies in grass pollen hay fever. J Immunol. 1979;122(6):2218-25.

42. Soderstrom L, Lilja G, Borres MP, Nilsson C. An explorative study of low levels of allergen-specific lgE and clinical allergy symptoms during early childhood. Allergy. 2011;66(8):1058-64. doi:10.1111/j.1398-9995.2011.02578.x.

43. Morokata T, Ishikawa J, Yamada T. Antigen dose defines T helper 1 and $T$ helper 2 responses in the lungs of C57BL/6 and BALB/C mice independently of splenic responses. Immunol Lett. 2000;72(2):119-26.

44. Riedl MA, Landaw EM, Saxon A, Diaz-Sanchez D. Initial high-dose nasal allergen exposure prevents allergic sensitization to a neoantigen. J Immunol. 2005; 174(11):7440-5.

45. Baroli B. Penetration of nanoparticles and nanomaterials in the skin: fiction or reality? J Pharm Sci. 2010;99(1):21-50. doi:10.1002/jps.21817.

46. Platts-Mills TA, Vaughan JW, Blumenthal K, Pollart Squillace S, Sporik RB. Serum IgG and IgG4 antibodies to Fel d 1 among children exposed to 20 microg Fel $d 1$ at home: relevance of a nonallergic modified Th2 response. Int Arch Allergy Immunol. 2001;124(1-3):126-9.

47. Matsui EC, Diette GB, Krop EJ, Aalberse RC, Smith AL, Curtin-Brosnan J, et al. Mouse allergen-specific immunoglobulin $\mathrm{G}$ and immunoglobulin $\mathrm{G} 4$ and allergic symptoms in immunoglobulin E-sensitized laboratory animal workers. Clin Exp Allergy. 2005:35(10):1347-53. doi:10.1111/j.1365-2222.2005.02331.x.

48. Jeal H, Draper A, Harris J, Taylor AN, Cullinan P, Jones M. Modified Th2 responses at high-dose exposures to allergen: using an occupational model. Am J Respir Crit Care Med. 2006;174(1):21-5. doi:10.1164/ rccm.200506-9640C.

49. Ilves M, Palomaki J, Vippola M, Lehto M, Savolainen K, Savinko T, et al. Topically applied $\mathrm{ZnO}$ nanoparticles suppress allergen induced skin inflammation but induce vigorous IgE production in the atopic dermatitis mouse model. Part Fibre Toxicol. 2014;11:38. doi:10.1186/s12989-014-0038-4.

50. Schilling K, Bradford B, Castelli D, Dufour E, Nash JF, Pape W, et al. Human safety review of "nano" titanium dioxide and zinc oxide. Photochem Photobiol Sci. 2010;9(4):495-509. doi:10.1039/b9pp00180h.

\section{Submit your next manuscript to BioMed Central and take full advantage of:}

- Convenient online submission

- Thorough peer review

- No space constraints or color figure charges

- Immediate publication on acceptance

- Inclusion in PubMed, CAS, Scopus and Google Scholar

- Research which is freely available for redistribution

Submit your manuscript at www.biomedcentral.com/submit 